\title{
HÄUFIG ANGEFÜHRTE HANDSCHRIFTEN
}

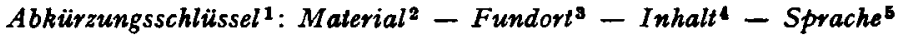 \\ 1QIsa DSIa ${ }^{a}$ Vollständige Jesajahandschrift aus $1 Q(\rightarrow 231)$ \\ 1QIs ${ }^{b}$ DSIb Unvollstăndige Jesajahandschrift aus $1 Q(\rightarrow 1364)$ \\ 1QpH DSH Habakukkommentar / Habakkuk Commentary / Commentaire \\ d'Habacuc aus 1Q $(\rightarrow 231)$ \\ $1 \mathrm{QS}[\boldsymbol{r k}]$ DSD Sektenschrift (-kanon, -rolle) / Manual of Discipline / Manuel \\ de Discipline aus $1 Q(\rightarrow 232)$ \\ 1QSa. b "Règle de la Congrégation* und "Recueil des Bénédictions" aus \\ $1 Q, 1 Q S$ zugehörig oder ähnlich $(\rightarrow \mathbf{8 5}, \mathrm{Nr} .28$ a. b) \\ 1QM[lhmh] DSW Der Krieg der Söhne des Lichts gegen die Söhne der Finsternis / \\ The War of the Sons of Light against the Sons of Darkness / \\ La Guerre des Fils de lumière contre les Fils de ténèbres aus \\ $1 Q(\rightarrow 1364)$ \\ $1 Q H$ [wdjwt] DST Hymnen aus $1 Q(\rightarrow 1364)$ \\ $1 \mathrm{QH}^{5}$ \\ Die 5 Hymnen aus 1361/1362 ("Hodajoth $A-E_{4}$ ) \\ $1 \mathrm{QIV}$ \\ DSL Aram. Genesismidrasch aus $1 Q(\rightarrow 85.1403$; nicht "Lamech- \\ buch" [1084. 85]). Teilveröffentlicht \\ cu3Q \\ 4QpNah \\ Kupferrollen aus $3 Q(\rightarrow 601)$. Unveröffentlicht \\ MurA \\ Nahumkommentar aus $4 Q(\rightarrow 1397)$ \\ MurB \\ MurC \\ XXII \\ $\mathbf{x B}$ \\ MirdA \\ Fragment in unbekannter Schrift aus Mur $(\rightarrow 147)$

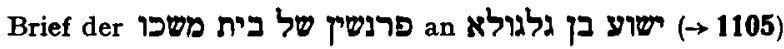

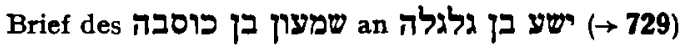 \\ Gr. Dodekapropheton unbekannten Fundorts $(\rightarrow 84)$ \\ Aramäischer Vertrag von 134 n. Chr. $\leftrightarrow$ 731) \\ CD \\ Christlich-palästinischer Brief aus Mird $(\rightarrow 730)$ \\ CDC Damaskusschrift, Sadokitisches Werk / Damascus Document, \\ Zadokite Fragments / Ecrit de Damas, Ecrit Sadocite aus der \\ Kairoer Geniza $(\rightarrow 930.891 .1215 .836)$ \\ PNash \\ Papyrus Nash $\rightarrow$ 612)
}

1 Nach J. T. MILIK, in: Qumran Cave I, Oxford 1955, 46f; R. DE VAUX, RB 60 (53), 87f; O. EISSFELDT, Einleitung in das AT, Tübingen ${ }^{2} 1956,799$ n. 1 ; W. H. BRowNLeE, NTSt $3(56 / 57), 13$ n. 1 . Ich benutze gekürzte Sigla.

Leder $=-$. Papyrus $=\mathrm{p}(\mathrm{ap})$. Kupfer $=\mathrm{cu}$. Ostrakon $=\mathrm{o}(\mathrm{s})$.

- $\mathrm{C}=$ Cairo Genizah. Sonst $\rightarrow$ Abkürzungsverzeichnis

- Bereits bekannte Werke: übliche Sigla; "Kommentaren« wird $\mathrm{P}[\mathrm{s̆}]$ vorgesetzt. Damaskusschrift: D. Neuentdecktes: Abkürzung des (vermutlichen) Originaltitels oder die halbfette Nummer der endgültigen Ausgabe. Hochbuchstaben unterscheiden verschiedene Mss. desselben Werkes. - Die Funde neuen Inhalts außerhalb $Q$ sind $\mathrm{m}$. W. noch ohne Sigla; ich bezeichne ihren Inhalt in der Reihenfolge des Erscheinens mit Großbuchstaben.

5 Hebraisch wird nicht bezeichnet.

- System der Jerusalemer American School of Oriental Research $(\rightarrow 231, X I)$. 
